\title{
Obtaining optical properties on demand
}

\author{
Reconfigurable metamaterials provide a flexible platform for nanophotonic technology
}

\section{By Nikolay I. Zheludev ${ }^{1,2}$}

W

ith the developments in nanotechnology that enable atoms, singly or in clumps, to be moved and arranged at will, we now have the capability of creating metamaterials that can display properties not found in naturally occurring media. Today lenses are being developed that image more sharply than glass lenses, and materials can be designed that make objects invisible or bend light beams into any chosen trajectory. We look here at the impact that metamaterials are having on photonics (see the figure).

Despite the achievements so far, photonic metamaterials cannot yet be used for many practical applications because of limitations associated with energy dissipation in the metals used to construct them. Alternatives are being sought in oxides and nitrides (1), topological insulators, and two-dimensional materials, which could offer improved plasmonic response, and in high-index dielectrics delivering resonant metamaterial properties with negligible losses (2). Planar phase and intensity holograms exploiting metamaterials with spatially variable characteristics may have potential for novel applications $(3,4)$. Metamaterials with zero dielectric permittivity (5) and topologically protected surface modes (6) promise new waveguide technologies; metamaterials with tailored hyperbolic dispersion (7) can enhance luminescence and improve optical gain. An emerging direction is to use metamaterials for optical computation (8) and thermal and radiation management.

The most remarkable recent development in this materials science is that we can now tune and switch metamaterial optical properties (9). However, today's challenge is not only to achieve homogeneous change of optical response across the entire volume of the metamaterial, but to develop the "on-demand" control of individual metamolecules in the material. By analogy with electronic random access memory, such structures are called "randomly accessible metamaterials." These

${ }^{1}$ Centre for Photonic Metamaterials, Optoelectronics Research Center, University of Southampton, Southampton S017 1BJ, UK. ${ }^{2}$ Centre for Disruptive Photonic Technologies, The Photonics Institute, Nanyang Technological University, Singapore 637371. E-mail:niz@orc.soton.ac.uk metamaterials will not only allow for modulation of light's intensity or phase, but will offer full active control of the wavefront of electromagnetic radiation, tailoring of the near field, and ultimately multichannel data processing. Developing randomly accessible photonic metamaterials is a challenge: The metamolecules would have to be subwavelength optical switches with a physical volume of about $10^{-19} \mathrm{~m}^{3}$. To have an impact on telecommunications technologies, such switches must also be fast and energy-efficient.

Do we have in sight physical processes that can sufficiently alter the optical properties of individual metamolecules such that they can change the phase and intensity of the transmitted and reflected light? Liquid crystal and digital micromirror spatial light modulators are well known, but they have pixels at least a few micrometers in size, too big for metamaterial applications, and their bandwidth is only a few tens of kilohertz at best, whereas electro-optical crystal modulators are fast but bulky. However, there are emerging technologies that can deliver not only metamolecular-level switching controlled by electric or magnetic signals, but also switching with light. These are nanooptomechanical, phase-change, and coherent control technologies.

Some exceptional opportunities are provided by nano-optomechanics that takes advantage of the changing balance of forces at the nanoscale. At the submicrometer dimensions of the metamolecules, electromagnetic forces compete with elastic forces and can thus be used to reconfigure the shape of individual metamolecules or their mutual arrangement. Structured semiconductor nanomembranes are the ideal platform for such nano-optomechanical reconfigurable metamaterials (10). These structures can be driven thermoelastically, electrically, and magnetically. They can also be reconfigured by light-induced forces between elements of illuminated metamolecules. Their nonlinear, switching, electro-optical and magneto-optical characteristics can surpass those of natural media by orders of magnitude. Moreover, the nanoscale metamaterial building

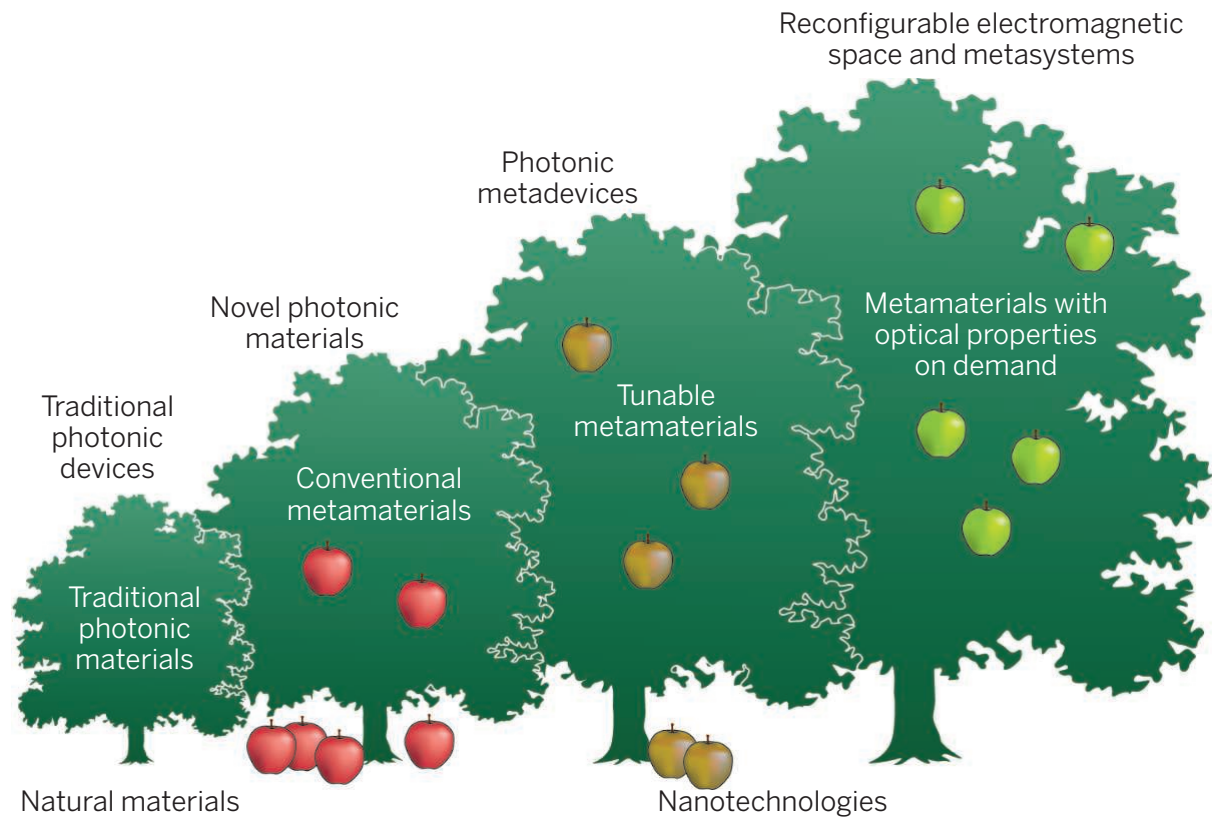

Mighty metamaterials forest. Metamaterials were first developed as artificial media structured on a size scale smaller than the wavelength of external stimuli. They showed novel, now well-understood electromagnetic properties, such as negative index of refraction or optical magnetism, allowing devices such as optical cloaks and superresolution lenses. Tunable, nonlinear, switchable, gain-assisted, sensor, and quantum metamaterials appeared and increased the potential for device integration of metamaterial technology. The coming challenge is to develop metamaterials with on-demand optical properties that may be independently controlled for every individual metamolecule of the nanostructure. 
blocks can be moved very fast, potentially offering gigahertz bandwidth switching. The first generation of randomly accessible reconfigurable metamaterials or nanomembranes, providing control in a single spatial dimension, have been realized and can function as refocusable lenses or dynamic diffraction gratings.

Phase change is another technology that can work for metamolecular-level switching. Initially developed for rewritable optical discs, it offers a mechanism for nonvolatile switching of optical properties within a nanoscale volume (11). This provides a new platform for creating optical components that are written, erased, and rewritten as two-dimensional binary or grayscale patterns into a film of chalcogenide glass using tailored trains of femtosecond pulses. Reconfigurable bichromatic and multifocus Fresnel zone plates, superoscillatory lenses with subwavelength focus, grayscale holograms, and a dielectric metamaterial with on-demand resonances have been demonstrated.

Another emerging technology for controlling and switching the manifestation of optical properties in metamaterials is coherent control. A highly absorbing plasmonic metamaterial film of subwavelength thickness that is placed in the node of a standing wave formed by counterpropagating control and signal waves will see zero electric field and so will not absorb the light. Any change in the phase or intensity of the control wave will distort the standing wave pattern and destroy the regime of zero absorption. This effect can underpin various forms of optical switching (12) operating down to the level of a few photons and with a modulation bandwidth up to 100 THz, presenting powerful opportunities for laser spectroscopies, image processing, and data handling in the locally coherent networks that are increasingly part of the mainstream telecommunications agenda.

REFERENCES

1. G. V. Naik, V. M. Shalaev, A. Boltasseva, Adv. Mater. 25 , 3264 (2013).

2. Y. Yang, I. I. Kravchenko, D. P. Briggs, J. Valentine, Nat. Commun. 5, 5753 (2014)

3. N. Yu. F. Capasso, Nat. Mater. 13,139 (2014)

4. S. Larouche, Y. J. Tsai, T. Tyler, N. M. Jokerst, D. R. Smith, Nat. Mater. 11, 450 (2012).

5. R. Maas, J. Parsons, N. Engheta, A. Polman, Nat. Photonics 7,907(2013)

6. A. B. Khanikaev et al., Nat. Mater. 12,233 (2013).

7. A. Poddubny, I. Iorsh, P. Belov, Y. Kivshar, Nat. Photonics 7, 948(2013).

8. A. Silva et al., Science 343,160 (2014).

9. N. I.Zheludev, Y. S. Kivshar, Nat. Mater. 11, 917 (2012).

10. J. Valente, J.Y. Ou, E. Plum, I. J. Youngs, N. I. Zheludev, Nat. Commun. 6, 7021(2015).

11. Q.Wang etal.,Appl. Phys. Lett. 104,121105 (2014).

12. X. Fang, K. F. MacDonald, N. I.Zheludev, Light Sci. Appl. 4 e292(2015)

INFECTIOUS DISEASES

\section{Overcoming neglect of kinetoplastid diseases}

\section{Drug development offers hope for controlling diseases that affect millions of people worldwide}

\section{By Graeme Bilbe}

1

$\mathrm{f}$ the 17 neglected tropical diseases listed by the World Health Organization (WHO) (1), three are caused by parasitic kinetoplastid protozoa: human African trypanosomiasis (HAT; also known as sleeping sickness), leishmaniasis, and Chagas disease. The three diseases are responsible for high mortality and morbidity among the world's poorest populations. Although these and other neglected diseases have received increased attention over the past decade, new drugs are still scarce: From 2000 to 2011, only $4 \%$ of new drugs and vaccines were registered for neglected diseases (2). However, the drug development pipeline, with sustained resources and research efforts, should see the delivery of new drugs for these diseases over the next decade.

KINETOPLASTID DISEASES. Transmitted by insects, these poverty-related infectious diseases are genetically highly diverse. They cause a spectrum of often chronic visceral and disfiguring skin diseases that can be fatal and that exact a high socioeconomic burden on patients and their families. Most cases occur in impoverished countries with poor health resources, but the diseases are also reemerging in Europe and the United States.

HAT is caused by Trypanosoma brucei (see the first figure). It is transmitted by the bite of an infected tsetse fly and is fatal without treatment. Active case detection and treatment have led to a fall in the number of cases, currently estimated at 20,000 , and sustained efforts are vital to ensure that the WHO's target to eliminate

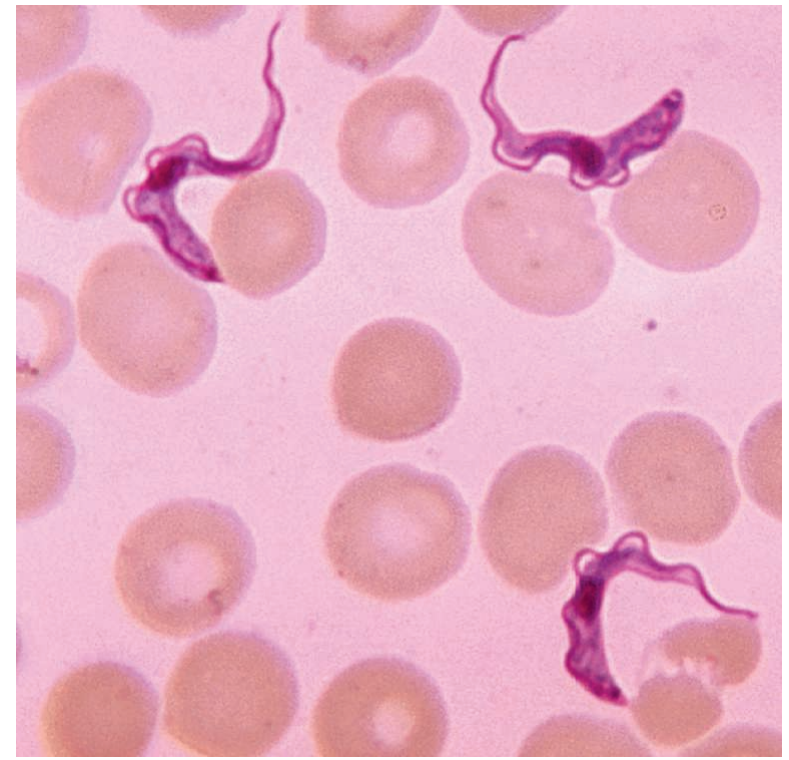

Trypanosoma sp. parasites in blood smear from a patient with African trypanosomiasis. The parasites are about 16 to $42 \mu \mathrm{m}$ long.
HAT as a public health problem by 2020 is achieved and maintained. The initial hemolymphatic phase of the disease generally goes undiagnosed without active surveillance.

Leishmaniasis is caused by Leishmania parasites that are transmitted by phlebotomine sandflies. Visceral leishmaniasis (fatal without treatment) and cutaneous leishmaniasis are the two most common forms of the disease, which is prevalent in 98 countries with 350 million people at risk. Leishmaniasis was long considered not to be a public health threat to high-income countries, but population increases, migration, and climate change may be spreading it, as highlighted by a recent serious outbreak in Madrid, Spain (3). Of even greater concern is the change in epidemiology of the more serious visceral form due to the spread of HIV (4).

Chagas disease is caused by T. cruzi parasites. An estimated 6 million to 7 million people worldwide are infected with the parasite. The disease is endemic in 21 countries of Latin America, where it causes more deaths than malaria, but can remain asymp- 
If you wish to distribute this article to others, you can order high-quality copies for your colleagues, clients, or customers by clicking here.

Permission to republish or repurpose articles or portions of articles can be obtained by following the guidelines here.

The following resources related to this article are available online at www.sciencemag.org (this information is current as of May 29, 2015 ):

Updated information and services, including high-resolution figures, can be found in the online version of this article at:

http://www.sciencemag.org/content/348/6238/973.full.html

This article cites 12 articles, 1 of which can be accessed free:

http://www.sciencemag.org/content/348/6238/973.full.html\#ref-list-1

This article appears in the following subject collections:

Physics

http://www.sciencemag.org/cgi/collection/physics 